\title{
WEIGHT FUNCTIONS ON EXTENSIONS OF THE COMPOUND MANUAL
}

\author{
by PATRICIA J. FRAZER, DAVID J. FOULIS, and CHARLES H. RANDALL
}

(Received 30 October, 1978)

1. Introduction. It has been shown ([8], [2], [1], [3], [9]) that a collection of physical operations or experiments can be represented by a nonempty set of nonempty sets satisfying certain technical conditions. Such a set $\mathscr{A}$ is called a manual. The operations in $\mathscr{A}$ are looked at as having no "before" and no "after", i.e., they are isolated in time. If we wish to look at connected sequences of operations-in particular, if we wish to condition by events in $\mathscr{A}$-we must look at the compound manual $\mathscr{A}^{c}$ whose elements represent compound operations built up from the operations in the base manual $\mathscr{A}$.

Central to the analysis of any given manual $\mathscr{A}$ are its weight functions, which are regarded as complete stochastic models of $\mathscr{A}$. It has been shown ([4]) that if $\mathscr{A}$ has a "lavish" supply of weights, then so does the compound manual $\mathscr{A}^{c}$. Our aim in this paper is to show that if $\mathscr{A}^{c}$ is extended in a seemingly natural way, in general all of these weight functions are lost. That is, there will be no stochastic models. possible on the resulting manual. Finally, we suggest one way in which this loss of weights may be remedied.

2. Terminology and symbolism. The definitions alone are presented here, as the motivation has been given in detail elsewhere. (See, for example, [8]). By a premanual $\mathscr{A}$, we mean a nonempty collection of nonempty sets. Elements of $\mathscr{A}$ are called operations. Each operation in $\mathscr{A}$ is itself a set, whose elements are the outcomes for that operation. $X=\bigcup \mathscr{A}$ will denote all the outcomes of $\mathscr{A}$, and

$$
\mathscr{E}(\mathscr{A})=\{D \subset X \mid \text { there is an } E \in \mathscr{A} \text { with } D \subset E\}
$$

will be called the set of events of $\mathscr{A}$. For $x, y \in X$, we say $x$ is orthogonal to $y$, and write $x \perp y$, if $x \neq y$ and there is an operation $E$ containing both $x$ and $y$. For $A \subset X, A^{\perp}$ will denote $\{x \in X \mid x \perp a$ for all $a \in A\}$, and $\{x\}^{\perp}$ will be written $x^{\perp}$ for $x \in X$. For $A, B \subset X$, $A \perp B$ will mean $A \subset B^{\perp}$. $A$ is called irredundant if for $E, F \in \mathscr{A}, E \subset F$ implies $E=F$. $A$ is called coherent if $A, B \in \mathscr{E}(\mathscr{A})$ with $A \perp B$ implies $A \cup B \in \mathscr{E}(\mathscr{A})$. An irredundant, coherent premanual is called a manual. A manual is called $\sigma$-coherent (respectively, completely coherent) if the union of any countable collection (respectively, any collection) of pairwise orthogonal events is again an event. The complete coherence of a manual $\mathscr{A}$, denoted $C(\mathscr{A})$, is the manual whose operations are precisely all the maximal orthogonal subsets of $X$. Clearly, $\mathscr{A} \subset C(\mathscr{A})$. A manual $\mathscr{A}$ is called Dacey if $E \in \mathscr{A}, x, y \in X$ with $E \subset x^{\perp} \cup y^{\perp}$ implies $x \perp y$. Most manuals considered in practice are Dacey.

By a weight function on $\mathscr{A}$, we mean a real-valued function $w$ defined on $X$ and satisfying

(1) $0 \leqslant w(x) \leqslant 1$ for all $x \in X$,

Glasgow Math. J. 21 (1980) 97-101. 
and

(2) for each $E \in \mathscr{A}$, the unordered sum $\sum_{x \in E} w(x)$ converges to 1 .

For $D \subset X$, we define $w(D)=\sum_{x \in D} w(x)$. The set of all weight functions on $\mathscr{A}$ will be denoted by $\Omega(\mathscr{A})$. A set $\mathscr{B}$ is called a submanual of $\mathscr{A}$ if $\mathscr{B} \subset \mathscr{A}$ and $\mathscr{B}$ is itself a manual. A submanual $\mathscr{B}$ of $\mathscr{A}$ is called an induced submanual of $\mathscr{A}$ if for $x, y \in \bigcup \mathscr{B}, x \perp y$ in $\mathscr{A}$ implies $x \perp y$ in $\mathscr{B}$. It is easily seen that if $\mathscr{B}$ is an induced submanual of $\mathscr{A}$ and if $w \in \Omega(\mathscr{A})$, then $w$ induces a weight on $\mathscr{B}$.

Each manual $\mathscr{A}$ is associated with its operational logic, which is denoted $\Pi(\mathscr{A})$. The elements of $\Pi(\mathscr{A})$ are $\{p(D) \mid D \in \mathscr{E}(\mathscr{A})\}$, where $p(D)$ is, by definition, the ordered pair $\left(D^{\perp \perp}, D^{\perp}\right)$. The partial ordering on $\Pi(\mathscr{A})$ is given by: $p\left(D_{1}\right) \leqslant p\left(D_{2}\right)$ if and only if $D_{1}^{\perp \perp} \subset D_{2}^{\perp \perp}$. It is known that a manual is Dacey if and only if its logic is an orthomodular poset. The structure of $\Pi(\mathscr{A})$ has been studied extensively ([9], [3]).

3. The compound manual. Let $S$ be the free semigroup on $X$, and let $X^{c}=S \cup\{1\}$, where $1 x=x=x 1$ for all $x \in X$. For $s \in S$, define the length of $s$, denoted $|s|$, to be $n$ if $s=x_{1} x_{2} \ldots x_{n}$ with $x_{i} \in X$ for $i=1, \ldots, n$. Define $|1|=0$. For $A \subset X^{c}$, define $|A|=$ $\max \{|a| \mid a \in A\}$. Call $A$ bounded if $|A|$ is finite. For $A, B \subset X^{c}$, let $A B=\{a b \mid a \in A$, $b \in B\} . \quad G$ will be called a direct successor of $E \subset X^{c}$ under the following conditions: $G=\bigcup_{e \in E} e F_{e}$, where for all $e$ in a nonempty subset $D$ of $E, F_{e}$ is some operation in $\mathscr{A}$, and for all $e \in E-D, F_{e}=1 . G$ is called a successor of $E$ if there is a finite sequence $E=G_{1}, G_{2}, \ldots, G_{n}=G$, where each $G_{i}, i=2, \ldots, n$, is a direct successor of $G_{i-1}$. The compound manual $\mathscr{A}^{\mathrm{c}}$ is defined to be all successors of 1 . The elements of $\mathscr{A}^{\mathrm{c}}$ are called compound operations.

It can be shown that $\mathscr{A}^{c}$ is a manual, with $\bigcup \mathscr{A}^{c}=X^{\mathrm{c}}$. For $a, b$ in $X^{\mathrm{c}}$, notice that $a \perp b$ in $\mathscr{A}^{c}$ if and only if there exist $c, d, e \in X^{c}$ and $x, y \in X$ such that $a=c x d, b=c y e$, and $x \perp y$ in $\mathscr{A}$. For $D \subset X^{c}, \quad a \in X^{c}$, define $a^{-1} D=\left\{c \in X^{c} \mid a c \in D\right\}$. Define $i(D)=$ $\left\{x \in X \mid x^{-1} D \neq \varnothing\right\}$. Call $E \subset X^{c}$ levelwise admissable if $i\left(a^{-1} E\right) \in \mathscr{E}(\mathscr{A})$ for every $a \in X^{\mathrm{c}}$. It can be shown that $G \in \mathscr{A}^{c}$ if and only if $G$ is a bounded, levelwise admissable, maximai orthogonal subset of $X^{c}$.

An analysis of $\mathscr{A}^{c}$ may be found in [3]. It is easily shown that $\mathscr{A}$ is Dacey if and only if $\mathscr{A}^{c}$ is Dacey. The connection between $\Omega(\mathscr{A})$ and $\Omega\left(\mathscr{A}^{c}\right)$ is investigated in [4]. It is shown there that weight functions on $\mathscr{A}$ induce weight functions on $\mathscr{A}^{c}$ and, furthermore, that every weight function on $\mathscr{A}^{c}$ arises in such a manner. It is also shown that $\Omega\left(\mathscr{A}^{c}\right)$ will inherit most of the properties that $\Omega(\mathscr{A})$ has. The fact that we have allowed only bounded compound operations is vital to each of these proofs. Unfortunately, if we allow unbounded compound operations-in particular, if we look at the complete coherence $C\left(\mathscr{A}^{c}\right)$ of $\mathscr{A}^{c}$-in most cases we lose all possible stochastic models.

4. Main result. It will now be shown that, under one very mild restriction, $C\left(\mathscr{A}^{c}\right)$ admits no weight functions. Theorem 1 is an adaptation of a theorem presented by $A$. Horn and A. Tarski in 1948 ([6]). 
THEOREM 1. Let $\mathscr{A}$ be a completely coherent, Dacey manual. Suppose $X=\bigcup \mathscr{A}$ has countable cardinality, and suppose further that $\Pi(\mathscr{A})$ is totally non-atomic. Then $\Omega(\mathscr{A})$ is empty.

Proof. Suppose there is a $w \in \Omega(\mathscr{A})$. We claim that for all $t>0$, for all $x \in X$, there is a $y \in x^{\perp \perp}$ such that $w(y)<t$. Suppose not. Then for all $y \in x^{\perp \perp}, w(y) \geqslant t>1 / n$ for some $n \in \mathbf{N}$. $\mathscr{A}$ is Dacey, so $\Pi(\mathscr{A})$ is an orthomodular poset. Furthermore, $\Pi(\mathscr{A})$ has no atoms, by assumption. Using these two facts, one can verify that there are nonempty events $D_{1}, D_{2}, \ldots, D_{n}$ in $\mathscr{A}$ such that $p(x)=p\left(D_{1}\right) \oplus p\left(D_{2}\right) \oplus \ldots \oplus p\left(D_{n}\right)$. For each $i=1, \ldots, n$, select a $y_{i} \in D_{i}$. Then $1 / n<w\left(y_{i}\right) \leqslant w\left(D_{i}\right)$ for $i=1, \ldots, n$, so $1=\sum_{i=1}^{n} 1 / n<\sum_{i=1}^{n} w\left(D_{i}\right)=$
$w(x)$, a contradiction.

Now, for each outcome $x \in X$, choose a $t_{x}>0$ such that $\sum_{x \in X} t_{x}=1$. Also, for each $x \in X$, by the claim, we may choose a $y_{x} \in x^{\perp \perp}$ with $w\left(y_{x}\right)<t_{x}$. Let $E$ be a maximal orthogonal subset of $\left\{y_{x} \mid x \in X\right\}$. By complete coherence, $E \in \mathscr{E}(\mathscr{A})$. However, $E \notin \mathscr{A}$ since $w(E) \leqslant \sum_{x \in X} w\left(y_{x}\right)<\sum_{x \in X} t_{x}=1$. Therefore, there is an $x \in E^{\perp}$, and hence $y_{x} \in x^{\perp \perp} \subset E^{\perp}$, a contradiction to the maximality of $E$.

THEOREM 2. Let $\mathscr{A}$ be any manual, $Q \in \mathscr{A}$ with $2 \leqslant \operatorname{card}(Q) \leqslant \kappa_{0}$. Let $Z=$ $\left\{q_{1} q_{2} \ldots q_{n} \mid q_{i} \in Q, n \in \mathbf{N}\right\}$. Let $\mathscr{B}$ be any $\sigma$-coherent induced submanual of $C\left(\mathscr{A}^{c}\right)$ such that $Z \subset \cup \mathscr{B}$. Then $\Omega(\mathscr{B})=\varnothing$.

Proof. Note that $Z \subset X^{c}$ and hence inherits an orthogonality relation from $\mathscr{A}^{c}$. Let $\mathscr{C}$ be the set of all maximal orthogonal subsets of $Z$.

Claim 1. $\mathscr{C}$ is an induced submanual of $\mathscr{B}$.

Proof. It is first shown that $\mathscr{C}$ is an induced submanual of $C\left(\mathscr{A}^{c}\right)$. Let $H \in \mathscr{C}$ Let $a \in X^{c}$. Suppose $i\left(a^{-1} H\right) \neq \varnothing$. Then $a \in Z$. It is clear that $i\left(a^{-1} H\right) \subset Q$. Suppose there is a $q \in Q$ with $q \notin i\left(a^{-1} H\right)$. Then $q \perp i\left(a^{-1} H\right)$ and hence $a q \perp H$, contradicting the maximality of $H$. Therefore, for all $a \in X^{\mathrm{c}}, i\left(a^{-1} H\right)=\varnothing$ or $i\left(a^{-1} H\right)=Q$. Now, suppose there is an $a \in X^{c}$ with $a \perp H$. We have $a=x_{1} x_{2} \ldots x_{n}$ for some $n$ and some $x_{i} \in X$. Since $a \notin H$, there is a minimal $j$ with $1 \leqslant j \leqslant n$ such that $x_{j} \notin i\left(\left(x_{1} \ldots x_{j-1}\right)^{-1} H\right)$. (If $j=1$, take $\left(x_{1} \ldots x_{j-1}\right)^{-1} H=H$. $)$ Since $a \perp H$, we have $i\left(\left(x_{1} \ldots x_{j-1}\right)^{-1} H\right) \neq \varnothing$ and $x_{i} \perp$ $i\left(\left(x_{1} \ldots x_{j-1}\right)^{-1} H\right)$. Thus, $x_{j} \perp Q$, a contradiction. Hence, $H$ is a maximal orthogonal subset of $X^{c}$, and we have $\mathscr{C} \subseteq C\left(\mathscr{A}^{c}\right)$. That $\mathscr{C}$ is then an induced submanual of $C\left(\mathscr{A}^{c}\right)$ follows immediately. Using the facts that $Z$ is countable (since $Q$ is), that $\mathscr{B}$ is $\sigma$-coherent, and that $Z \subset \cup \mathscr{B}$, it is seen that $\mathscr{C} \subset \mathscr{B}$. It is then easily verified that $\mathscr{C}$ is, in fact, an induced submanual of $\mathscr{B}$.

Therefore, if suffices to show that $\mathscr{C}$ has no weight functions to prove the theorem.

Claim 2. $\mathscr{C}$ is Dacey.

Proof. Suppose $z_{1}, z_{2} \in Z, H \in \mathscr{C}$ with $H \subset z_{1}^{1} \cup z_{2}^{\perp}$. Clearly, $z_{1} \neq z_{2}$. Suppose $z_{1} \perp z_{2}$ fails. This implies that $z_{1}=q_{1} q_{2} \ldots q_{r}$ and $z_{2}=q_{1} q_{2} \ldots q_{s}$ for some $r \neq s$ and some $q_{i} \in Q$. Without loss of generality, suppose $s<r$. But then $z_{2}^{\perp} \subset z_{1}^{\perp}$, and we have $H \subset z_{1}^{\perp}$, a contradiction. 


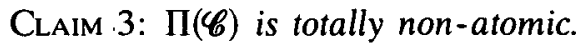

Proof. Suppose $p(D)$ is an atom in $\Pi(\mathscr{C})$. Then it is clear that $D=\{a\}$ for some $a \in Z$. Let $q \in Q$. Since $2 \leqslant \operatorname{card}(Q)$, there is a $q^{\prime} \in Q$ with $q \perp q^{\prime}$. Therefore, $0<p(a q)<p(a)$ in $\Pi(\mathscr{C})$, since $a q^{\prime} \perp a q$ but $a q^{\prime} \perp a$ fails. This supplies our contradiction, and the claim is proved.

By definition, $Z$ is countable and $\mathscr{C}$ is completely coherent. Therefore, $\mathscr{C}$ satisfies the hypotheses of Theorem 1 , and hence has no weights.

COROLlary. Let $\mathscr{A}$ be any manual $Q \in \mathscr{A}$ with $2 \leqslant \operatorname{card}(Q) \leqslant \aleph_{0}$. Then there are no weight functions on $C\left(\mathscr{A}^{c}\right)$.

5. The finitary embedding of $C\left(\mathscr{A}^{c}\right)$. A manual $\mathscr{A}$ is called hereditary provided that, for every finite non-empty subset $\mathcal{M}$ of $\mathscr{A}$, the set $\{E \in \mathscr{A} \mid E \subset \cup \mathcal{M}\}$ is an induced submanual of $\mathscr{A}$. A hereditary Dacey manual (that is, a manual which is both hereditary and Dacey) will be called an HD manual. It is known that a manual is HD if and only if it does not admit four distinct outcomes $a, b, c, d$ such that $a \perp b, b \perp c, c \perp d, a \notin c^{\perp}, a \notin d^{\perp}$, and $b \notin d^{\perp}$. One can verify that $\mathscr{A}$ is HD if and only if $\mathscr{A}^{c}$ is $H D$, and this is so if and only if $C\left(\mathscr{A}^{c}\right)$ is $H D$. The logic of an $H D$ manual is an orthomodular lattice. As many formulations of quantum mechanics require that the so-called "quantum logic" be an orthomodular lattice (see, e.g., Mackey [7]), these manuals are of particular interest in applications to quantum physics.

A manual $\mathscr{A}$ is said to admit a full set $\Delta$ of weight functions if $x, y \in X$ with $x$ not orthogonal to $y$ in $\mathscr{A}$ implies there is a $w \in \Delta$ with $w(x)+w(y)>1$. Of particular significance are those manuals which admit full sets of dispersion-free weights (i.e. weights assuming only the values 0 and 1 ). In this case, other weight functions may arise as convex linear combinations of the dispersion-free weights, indicating a possible way in which "hidden variables" may be present.

A scattered set, in a manual $\mathscr{A}$ is a set $S \subset X$ such that for each pair $s, t \in S, s \perp t$ fails. A manual is called finitary if every operation intersects every maximal scattered set. It is easily shown that every finitary manual admits a full set of dispersion-free weights.

Marie Gaudard and Robert Weaver have shown in [5] that every HD manual may be embedded in a finitary HD manual. This new manual is called the finitary completion. We have shown that if the base manual $\mathscr{A}$ is $\mathrm{HD}$, then $C\left(\mathscr{A}^{c}\right)$ will also be HD but may admit no weights. Therefore, we wish to examine the finitary completion of $C\left(\mathscr{A}^{c}\right)$.

Consider the following example. Suppose $\mathscr{A}$ consists of a single operation: to flip a coin and record the outcome. That is, $\mathscr{A}=\{\{t, h\}\}$. For each $n \in \mathbf{N}$ define $E_{n}=$ $\left\{t^{i-1} h \mid i=1, \ldots, n\right\} \cup\left\{t^{n}\right\} .\left(t^{0} h=h\right)$ Then each $E_{n} \in \mathscr{A}^{c}$ and $E_{n}$ is the operation "flip a coin $n$ times or until a head is observed, whichever comes first". The set $E=$ $\left\{t^{i-1} h \mid i=1,2,3, \ldots\right\}$, i.e., "flip a coin until a head is observed", is an operation in $C\left(\mathbb{A}^{c}\right)$ but not in $\mathscr{A}^{c}$ as it is unbounded. Thus, there are no weight functions on $C\left(\mathscr{A}^{c}\right)$ to accommodate this operation. This difficulty is due to the absence of the "in-principle" outcome $t^{\infty}$. 
If the base manual $\mathscr{A}$ is completely coherent and finitary HD, then the finitary completion of $C\left(\mathscr{A}^{c}\right)$ can be given explicitly as follows. Adjoin all infinite strings (maps $\mathbf{N} \rightarrow X$ ) to $X^{c}$. If $g$ is such an infinite string, say $g=x_{1} x_{2} x_{3} \ldots$, let $g_{n}$ denote $x_{1} \ldots x_{n}$. Extend the orthogonality of $\mathscr{A}^{c}$ to these new "outcomes" as follows. For $a \in X^{c}, g$ an infinite string, define $a \perp g$ if and only if $a \perp g_{n}$ for some $n$. Likewise, for two intinite strings $g, h$, define $g \perp h$ if and only if $g_{n} \perp h_{n}$ for some $n$. Then, a difficult but unilluminating proof shows that the finitary completion of $C\left(\mathscr{A}^{c}\right)$ is precisely all maximal orthogonal subsets of this extended outcome set.

Hence, if outcomes of infinite length are adjoined, we obtain an extension of the compound manual which has a full set of dispersion-free weight functions.

6. Conclusion. The restriction that $\mathscr{A}^{c}$ contain only bounded operations may initially appear to be a matter of choice; in some sense, the bounded operations are considered more do-able. This paper presents a much stronger argument for the restriction; without it, in most interesting cases all stochastic models are lost.

Furthermore, we have observed that if the base manual $\mathscr{A}$ is $\mathrm{HD}$, then the compound manual along with all unbounded operations may be embedded in a manual with a full set of dispersion-free weights. If $\mathscr{A}$ satisfies certain other restrictions, this embedding may be defined explicitly in terms of infinite sequences in $X$.

Thus the answer at this point seems to be that if unbounded operations are required, it appears necessary to include unbounded outcomes as well.

\section{REFERENCES}

1. D. J. Foulis and C. H. Randall, Operational statistics I, Basic Concepts, J. Mathematical. Phys. 13 (1972), 1667-1675. 81-111.

2. D. J. Foulis and C. H. Randäll, Empirical logic and quantum mechanics, Synthese 29 (1974),

3. D. J. Foulis and C. H. Randall. The empirical logic approach to the physical sciences, in Foundations of quantum mechanics and ordered linear spaces, (Springer-Verlag, 1974).

4. D. J. Foulis and C. H. Randall. The stability of pure weights under conditioning, Glasgow Math. J. 15 (1974), 5-12.

5. M. A. Gaudard and R. J. Weaver, Finitary embeddings of certain generalized sample spaces, Trans. Amer. Math. Soc. 207 (1975), 293-307.

6. A. Horn and A. Tarski, Measures in Boolean Algebras, Trans. Amer. Math. Soc. 64 (1948), 467-497.

7. G. W. Mackey. The mathematical foundations of quantum mechanics, (Benjamin, 1963).

8. C. H. Randall and D. J. Foulis, The operational approach to quantum mechanics, in Hooker, C. A. (Ed.), The logico-algebraic approach to quantum mechanics III, (D. Reidel Publishing Co., 1977).

9. C. H. Randall and D. J. Foulis. A mathematical setting for inductive reasoning, in Hooker, C. A. (Ed.), Foundations of probability theory, statistical inference, and statistical theories of science III, (D. Reidel Publishing Co., 1976), 169-205.

UNIVERSITY OF MASSACHUSETTS

AMHerst

Massachusetts 01002 